\title{
Influence of externally applied magnetic perturbations on neoclassical tearing modes at ASDEX Upgrade
}

\author{
$\underline{\text { S. Fietz }}^{1}$, A. Bergmann ${ }^{1}$, I. Classen ${ }^{2}$, M. Maraschek ${ }^{1}$, M. \\ Garcia-Muñoz $^{3}$, W. Suttrop ${ }^{1}$, H. Zohm ${ }^{1}$ and the ASDEX \\ Upgrade Team \\ ${ }^{1}$ Max Planck Institute for Plasma Physics, Boltzmannstr. 2, 85748 Garching, \\ Germany \\ ${ }^{2}$ FOM Institute DIFFER-Dutch Institute for Fundamental Energy Research, \\ Association EURATOM-FOM, Nieuwegein, The Netherlands \\ ${ }^{3}$ Faculty of Physics, FAMN Dpt.,University of Seville, Seville, Spain \\ E-mail: sina.fietz@ipp.mpg.de
}

\begin{abstract}
.
The influence of externally applied magnetic perturbations (MPs) on neoclassical tearing modes (NTM) and the plasma rotation in general is investigated at the ASDEX Upgrade tokamak (AUG). The low $n$ resonant components of the applied field exert local torques and influence the stability of NTMs. The non-resonant components of the error field do not influence MHD modes directly but slow down the plasma rotation globally due to a neoclassical toroidal viscous torque (NTV). Both components slow down the plasma rotation, which in consequence increases the probability for the appearance of locked modes. To investigate the impact of externally applied MPs on already existing modes and the influence on the rotation profile, experimental observations are compared to modelling results. The model used here solves a coupled equation system that includes the Rutherford equation and the equation of motion, taking into account the resonant effects and the resistive wall. It is shown that the NTV torque can be neglected in this modelling. To match the experimental frequency evolution of the mode the MP field strength at the resonant surface has to be increased compared to the vacuum approximation. This leads to an overestimation of the stabilising effect on the NTMs. The reconstruction of the entire rotation profile via the equation of motion including radial dependencies, confirms that the NTV is negligibly small and that small resonant torques at different resonant surfaces have the same effect than one large one. This modelling suggests that in the experiment resonant torques at different surfaces are acting and slowing down the plasma rotation requiring a smaller torque at the specific resonant surface of the NTM. This additionally removes the overestimated influence on the island stability, whereas the braking of the island's rotation is caused by the sum of all torques. Consequently, to describe the effect of MPs on the evolution of one island, all other islands and the corresponding torques must also be taken into account.
\end{abstract}

PACS numbers: $52.55 \mathrm{Fa}, 52.55 . \mathrm{Tn}$

Submitted to: Nuclear Fusion 


\section{Introduction}

Externally applied magnetic perturbations (MPs) are of great interest for the operation of future fusion devices. They are used for active MHD control, especially for the mitigation of edge localised modes (ELMs) $[1,2,3]$. Additionally also the active stabilisation of resistive wall modes [4] and neoclassical tearing modes (NTMs) [5] has already been shown. These are some reasons why magnetic perturbation coils are also planned to be part of the ITER design. However, these actively applied 3D fields have typically also negative side-effects such as density pump out [6, 7, 8, 9] and an increase of fast ion losses $[9,10]$, but additionally they can act on the plasma stability in an unfavourable way.

The applied resonant MP fields can penetrate the plasma and provoke magnetic reconnection at the corresponding resonant surfaces, which is accompanied with the generation of a magnetic islands there. These small islands can act as seed island for an NTM $[1,11,12]$. Static resonant MPs can hence produce a locked mode which in most of the cases even leads to a disruption. Also pre-existing rotating modes can interact with the resonant MP fields $[1,5]$. They can be slowed down and even locked to the MP field. The non-resonant components of the error field do not influence MHD modes directly but instead induce a global torque, the so called neoclassical toroidal viscous (NTV) torque, and hence also influence the critical error field strength for the appearance of locked modes [13]. As it is tried to avoid NTMs in general, or at least the locking of NTMs, because of the negative effect on the plasma confinement, the application of MP coils may be accompanied with some problems.

To study the influence of such externally applied magnetic perturbations, the ASDEX Upgrade tokamak has recently been equipped with a set of 16 in-vessel saddle coils [14]. They are installed at the low field side in two rows above and below the mid plane. This set of coils, so called B-coils, enables the generation of MPs with a toroidal mode number of $n \leq 4$. These low $n$ mode spectra are perfectly suitable to study the influence of the MPs on the $(m, n)=(3,2)$ and $(2,1)$ NTMs $(m$ the poloidal and $n$ the toroidal mode number) which are the most common and confinement degrading NTMs in AUG.

NTMs are resistive MHD instabilities which are driven by a loss of bootstrap current inside the island. Various stabilising and destabilising mechanisms contribute to the width evolution of NTMs, described by the modified Rutherford equation [15, 16, 17]. Once a sufficiently large seed island is generated, the destabilising effect of the bootstrap current defect and the stabilising effect of the equilibrium current profile $\left(\Delta^{\prime}\right)$ are the main drivers of an NTM. For small islands further stabilising and destabilising effects like transport effects [18], the ion polarisation current [19], the toroidicity and shaping $[20,21,22,23]$ additionally alter the island stability.

In this paper two different discharges are presented, where an influence of the B-coils on NTMs is observed. It is shown that the influence of the B-coils on the mode frequency and the island width evolution can be modelled using a coupled equation system based on a modified Rutherford equation and the equation of motion [24]. However, to best 
model the evolution of the island frequency the amplitude of the resonant MP field component had to be increased compared to its vacuum value. At the same time the stabilising influence on the island amplitude had to be reduced. The torque balance equation is calculated including a viscous torque, the $(\vec{j} \times \vec{B})$ resonant torque induced by the B-coils and the resistive wall torque. Also the influence of the non-resonant NTV torque on the plasma rotation is estimated. Experimental observations and the comparison with modelling results show that the resonant effects play the dominant role and the non-resonant contributions of the B-coils, like the NTV [25, 26], seem to be rather negligible. Further it is shown that to fully describe the evolution of one island, all torques at different resonant surfaces have to be taken into account.

\section{Basic model}

Magnetic islands can be strongly influenced by externally applied MP fields which are produced by currents flowing in external coils. The resonant components directly influence the island stability and additionally induce a local torque at the resonant surfaces. The non-resonant components do not influence the island directly but, due to a global braking torque of the whole plasma, also the island is slowed down towards a neoclassical toroidal offset frequency $\Omega_{\mathrm{NC}}^{*}$.

Resonant effects. The resonant components of the perturbation field exhibit a gradient discontinuity in the perturbed helical poloidal flux at the resonant surfaces, which implies the existence of a helical current perturbation being present at these surfaces [24]. Without conducting plasma the resonant MP field components, result in island structures in the vacuum magnetic field, as illustrated in figure 1. The vacuum magnetic field is calculated as a superposition of the equilibrium and the MP field.

In the presence of a conducting plasma a resonant component of the MP field induces a helical shielding current at the corresponding resonant surface, which suppresses MP field driven magnetic reconnection. Hence, this helical current generated at the resonant surface leads to a shielding of the perturbation field. However, this shielding current influences a magnetic island at the corresponding resonant surface in two ways: it modifies the island width evolution due to an influence on the island stability, which can be described by a modified Rutherford equation. This interaction takes place for the $\cos (\Delta \phi)$ component of the helical shielding current. $\Delta \phi$ is the angular phase difference of the O-points of the magnetic and the vacuum island. On the other hand the $\sin (\Delta \phi)$ component of the current causes a $(\vec{j} \times \vec{B})$ torque which is acting on the island in order to adapt the rotation frequency of the island to the rotation frequency of the perturbation field.

This theory assumes full shielding of the MP field and takes into account the plasma rotation via the dependence on the phase difference of the island and the perturbation field $\Delta \phi$. This regime of strong shielding of the MP field is followed by "full reconnection" $[24,27]$ and a concomitant sudden drop of plasma rotation once the 
MP field exceeds a critical value.

The perturbed magnetic flux $\psi_{\mathrm{vac}}$ at a resonant surface can be parametrised using the vacuum field approximation via the size of the vacuum island $W_{\text {vac }}$ in a cylindric geometry.

$$
W_{\text {vac }}=4\left(\frac{R_{0} q}{B_{\text {tor }}}\right)^{\frac{1}{2}}\left|\frac{\psi_{\text {vac }}}{q^{\prime} / q r_{\text {res }}}\right|^{\frac{1}{2}},
$$

It can also be linked to the perturbed radial magnetic field $\left(b_{\mathrm{r}, \mathrm{vac}}=-\left(m_{\mathrm{pol}} \psi_{\text {vac }}\right) / r\right)$. Hence $W_{\text {vac }}$ is a good measure for the amplitude of the magnetic perturbation field. The vacuum magnetic field approximation, which results from the superposition of the unperturbed magnetic field with the vacuum perturbation of the field coils for two ASDEX Upgrade discharges is shown in figure 1. The vacuum islands due to the applied $n=1$ and $n=2$ error fields are clearly visible, especially at the $q=2$ and $q=3 / 2$ surface, respectively. Since the theory is developed for cylindrical plasmas, the quantities taken
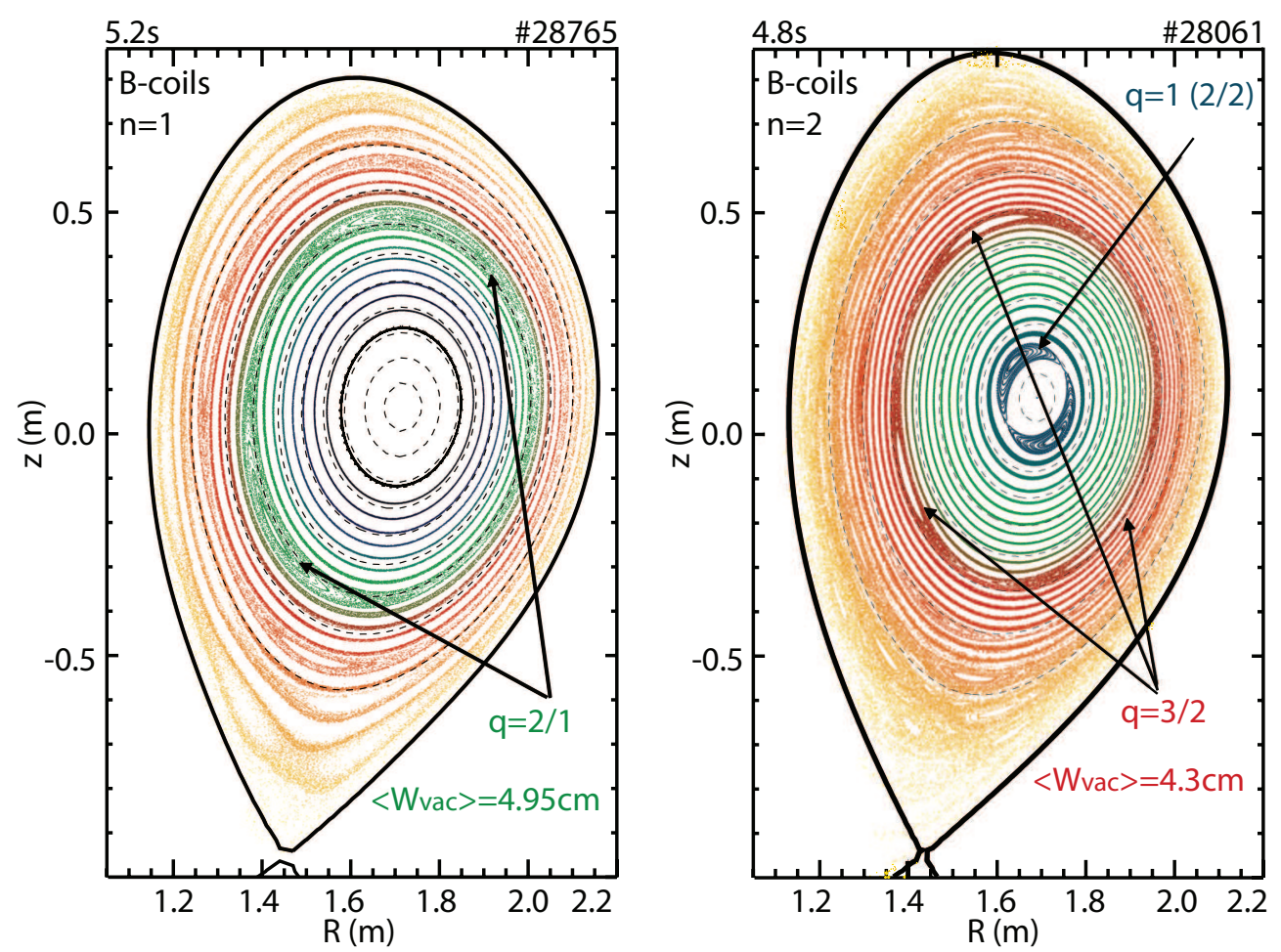

Figure 1. Poincare plot of the vacuum magnetic field in the poloidal plane for the discharge $\# 28765\left(B_{\text {tor }}=2.5 \mathrm{~T}, I_{\mathrm{p}}=0.8 \mathrm{MA}, I_{\mathrm{B}-\text { coils }}=5215 \mathrm{kA}, q_{95} \approx 5.2\right)$ and $\# 28061\left(B_{\text {tor }}=1.73 \mathrm{~T}, I_{\mathrm{p}}=0.8 \mathrm{MA}, I_{\mathrm{B}-\text { coils }}=5750 \mathrm{kA}, q_{95} \approx 3.8\right)$. The B-coils have been used with an $n=1$ and $n=2$ configuration which leads to pronounced vacuum islands at the $\mathrm{q}=2$ and the $\mathrm{q}=3 / 2$ surface respectively. Also further vacuum islands at other surfaces are present.

from the experiment with toroidal geometry have to be adjusted. The induced vacuum island width for example is not constant on a flux surface. Therefore the flux surface averaged vacuum island width is used for the calculation, corresponding to the flux 
surface averaged resonant component of the perturbation field at the resonant surface in real AUG geometry.

Non-resonant effects. The non-resonant components of the externally applied MP fields can affect the plasma rotation and hence the mode frequency via the NTV torque. These non-resonant components of the error field lead to a deformation of the former toroidally symmetric plasma equilibrium and result in a nonaxisymmetric 3D equilibrium. Due to this symmetry breaking trapped particles drift radially outward. This results in a radial particle flux $\Gamma$ and a concomitant global toroidal plasma braking. This phenomena is called neoclassical toroidal viscous torque (NTV). The NTV changes plasma rotation towards an offset rotation where the net drift of particles is zero, hence contributes to the plasma rotation damping. The influence of the NTV torque on the plasma stability has already been studied in several devices [13, 28, 29, 30, 30, 31]. Compared to the resonant $(\vec{j} \times \vec{B})$ torque which acts only locally, the non-resonant components affect a global torque at different flux surfaces through the NTV which also influence the resonant surfaces. Contrary to the resonant torque the NTV induced torque however does not influence the island stability. To calculate the NTV analytically the toroidal momentum equation, which connects the radial particle transport and the toroidal forces, has to be solved. Hence, the NTV can be calculated from the toroidal component $\left(\vec{e}_{\phi}\right)$ of the momentum equation and the flux friction relation $\left\langle\vec{e}_{\phi} \nabla \cdot \pi_{\mathrm{i}}\right\rangle=Z_{\mathrm{i}} e \Gamma_{\mathrm{i}}$ with $\pi_{\mathrm{i}}$ being the ion viscous stress tensor, $e$ the elementary electric charge and $Z_{\mathrm{i}}$ the atomic number . The resulting formulae are described in $[32,25,26]$, which can be used to calculate the NTV induced by non helical MPs in a real non cylindric geometry.

\subsection{Island stability}

The evolution of the island width can be described by the modified Rutherford equation $[15,16,17]$. The stability of big islands, like in the investigated two cases, can mainly be described by the stability of the equilibrium current profile $\left(\Delta^{\prime}\right)$ and the loss of bootstrap current $\Delta_{\text {bs }}$ inside the island due to the flattening of pressure over the island region.

$$
\begin{aligned}
0.82 \frac{\tau_{R}}{r_{\text {res }}} \frac{d W}{d t} & =\Delta^{\prime} r_{\text {res }}+\Delta_{\text {bs }} r_{\text {res }}+\Delta_{\text {ext }} r_{\text {res }} \\
\Delta_{\mathrm{BS}} & =\frac{c_{\mathrm{BS}}}{W} \\
\Delta_{\text {ext }} & =\frac{2 m}{r_{\text {res }}}\left(\frac{W_{\text {vac }}}{W}\right)^{2} \cos (\Delta \phi)
\end{aligned}
$$

The parameter $r_{\text {res }}$ is the radius of the rational surface and $\tau_{\mathrm{R}}=\mu_{0} r_{\text {res }}^{2} /(1.22 \eta)$ the resistive time-scale.

For simplification, in the following an approximation for the $\Delta^{\prime}$-term and the contribution of the bootstrap current (bs) defect $\Delta_{\mathrm{bs}}$ is used. The equilibrium current profile is assumed to be stabilising and $\Delta^{\prime}$ approximated via $-m / r_{\text {res }}$. The destabilising contribution of $\Delta_{\text {bs }}$ decreases with increasing island width. Additionally it is assumed that the islands are saturated at a constant island size $(d W / d t=0)$ before switching 
on the B-coils. Hence the fit parameter $c_{\mathrm{bs}}$ is introduced to fulfil $d W / d t=0$ before switching on the B-coils $\left(\Delta_{\text {ext }}=0\right)$. The saturated island size $W_{\text {sat }}$ is determined from the experiment. The dependence of $W_{\text {sat }}$ on $\Delta^{\prime}$ is discussed in [33]. During the application of the MP field the island size is not changing significantly in both investigated cases, therefore the contribution of the bootstrap current defect, hence $c_{\mathrm{bs}}$, is assumed to be constant. Additionally, the effect of the resonant component of the external perturbation field [24] on the island width is included via $\Delta_{\text {ext }}$.

The influence of the MP field on the island width evolution varies with the angular phase difference between the O-points of the vacuum island and the magnetic island. In other words it depends on the position of the magnetic island with respect to the orientation of the MP field. Additionally the contribution of the MP field to the island with evolution is smaller for larger islands. We also investigated the influence of the stabilising effect of the wall [24] on the island width evolution, but this effect is small for the investigated discharges. In consequence it has not been taken into account in the analysis of the island growth in the following investigations. In both investigated cases the islands have a significant size, even during mode locking (compare figure 2). Therefore polarisation current effects and further small island effects can be neglected in the modelling.

\subsection{Equation of motion}

The evolution of the plasma rotation is described by the equation of motion. Due to poloidal flow damping the poloidal ion rotation is small in the plasma centre compared to the toroidal ion rotation velocity $[34,35]$. Hence it can be neglected and the plasma rotation is given only by its toroidal component. The equation of motion can be written as

$$
I \frac{d \omega}{d t}=\sum T_{\Phi}=T_{\mathrm{vs}}+T_{\mathrm{jxB}}+T_{\mathrm{rw}}+T_{\mathrm{NTV}}
$$

with $I$ representing the corresponding moment of inertia and $\omega$ the toroidal rotation frequency, defined as toroidal rotation velocity $v$ divided by the corresponding plasma radius $r(\omega=v(r) / r) . \quad r$ is a radial label according to the plasma radius which goes from the magnetic axis $R_{0}$ to the plasma separatrix.

In equation (5) the toroidal components of a viscous torque $T_{\mathrm{vs}}$ [24], the electromagnetic torque due to the external resonant perturbation field $T_{\mathrm{jxB}}[24]$, the resistive wall $T_{\mathrm{rw}}$ [36] and the torque due to NTV are included.

$$
\begin{aligned}
& \left.T_{\mathrm{vs}}\right|_{d V}=4 \pi^{2} R_{0}\left[\left(D_{\Phi} \rho r R_{0}^{2}\right) \frac{\partial \omega}{\partial r}\right]_{r-d r / 2}^{r+d r / 2} d r \approx-A \frac{1}{n}\left(\omega-\omega_{0}\right) \\
& T_{\phi, \mathrm{jxB}}=-4 \pi^{2} \frac{m n}{\mu_{0}} C^{2} W_{\mathrm{vac}}^{2} W^{2} \sin (\Delta \phi) \\
& T_{\phi, \mathrm{rw}}=-4 \pi^{2} R_{0}^{2} \frac{m^{2}}{\mu_{0} r_{\mathrm{res}}}\left(\frac{r_{\mathrm{res}}}{r_{\mathrm{W}}}\right)^{2 m} \frac{\left(\omega \tau_{\mathrm{W}}\right)}{1+\left(\omega \tau_{\mathrm{W}}\right)^{2}} C^{2} W^{4} \frac{1}{\sqrt{(\epsilon / q)^{2}+1}}
\end{aligned}
$$




$$
\begin{aligned}
& \text { with } C=\frac{r_{\mathrm{res}} B_{\text {tor }}\left|q^{\prime}\right|}{16 q^{2} R_{0}} \\
& T_{\mathrm{NTV}}=-\rho \mu_{\|} I_{\lambda}\left(\left\langle R^{2} \omega\right\rangle-\left\langle R^{2} \Omega_{N C}^{*}\right\rangle\right),
\end{aligned}
$$

with $\mu_{0}$ the magnetic constant, $\tau_{\mathrm{W}}$ the resistive time scale of the wall (50 ms at AUG) and $\epsilon$ the inverse aspect ratio at the resonant surface.

The plasma cross-sections (referred to as 'plasma shells' with volume $d V$ and width $d r$ ) are radially coupled via a perpendicular plasma viscosity. This gives rise to a viscous torque (eq. (d6)), where the toroidal component can be described according to $[24,37]$ with $\mathrm{D}$ the momentum diffusion coefficient and the mass density. The product of both, $D_{\phi}$ and $\rho$, is proportional to the perpendicular viscosity. A radial gradient in the rotation profile gives rise to a restoring torque between the different plasma shells along $r$ with the Volume $d V=V(r+d r / 2)-V(r-d r / 2)$. For a large island, which is not allowed to 'slip' through the plasma, only the deviation from its natural rotation frequency $\omega_{0}=\left(v_{0}\left(r_{\text {res }}\right) / r_{\text {res }}\right)$ is important. The natural frequency is defined as the island rotation in a steady-state situation where the global viscous and NBI input torque balance each other. Every deviation of $\omega$ from $\omega_{0}$ is opposed by the perpendicular viscosity which gives rise to the corresponding restoring torque. The torque can then be derived from the rightmost term in eq. 6 , assuming that changes in the mode rotation are caused by changes in the toroidal plasma rotation [37]. The parameter $A$ depends on viscosity and properties of the natural velocity profile. The external toroidal plasma rotation is mainly determined by the NBI input torque and for example by the externally induced NTV effects. As discussed in [38] the inertia term and the viscous term depend on the size of the modulation frequency due to the externally applied perturbation field. In the cases discussed within this paper the viscous torque is directly determined from the experiment whereas inertia effects are assumed to be mainly localised in the island region due to the significant size of the islands. The local resonant torque induced by the B-coils (eq. $(7))$ is proportional to the $\sin (\Delta \phi)$ component of the perturbation field and increases with increasing island size. The resistive wall torque (eq. (8)) is mainly important for slowly rotating islands, especially in the phases shortly before mode locking. It can be neglected for modes which are rotating at more than $1 \mathrm{kHz}$, due to its dependence on $\omega$. The NTV torque (eq. (10)) acts on the plasma rotation and hence via the viscous torque influences the island rotation. The NTV in the usually dominant banana regime depends on the toroidal viscosity frequency $\mu_{\|}$, the pitch angle integral $I_{\lambda}$ and the difference between the rotation frequency $\omega$ and the neoclassical offset frequency $\left\langle\Omega_{N C}^{*}\right\rangle \approx \frac{c_{p}+c_{t}}{e Z_{i}} \frac{d T_{\mathrm{i}}}{d \psi}$. For static MPs the rotation frequency $\Omega_{N C}^{*}$ is in countercurrent direction, which results in a damping of the plasma rotation. The derivation of the NTV torque in a non cylindric geometry is quite complicated. As will be shown in section 6 the NTV is small compared to the resonant contribution and in consequence it can be neglected in the modelling of the island rotation in section 4 .

Equation (5) can be evaluated locally, e.g. at the position of an island, hence $\omega$ corresponds to the island rotation frequency. This delivers the local mode frequency evolution and thus all torques have to be taken at the resonant surface. Additionally 
also the evolution of the entire rotation profile can be calculated, accounting for the radial dependence of all torques and a constant momentum diffusion coefficient $D_{\phi}$.
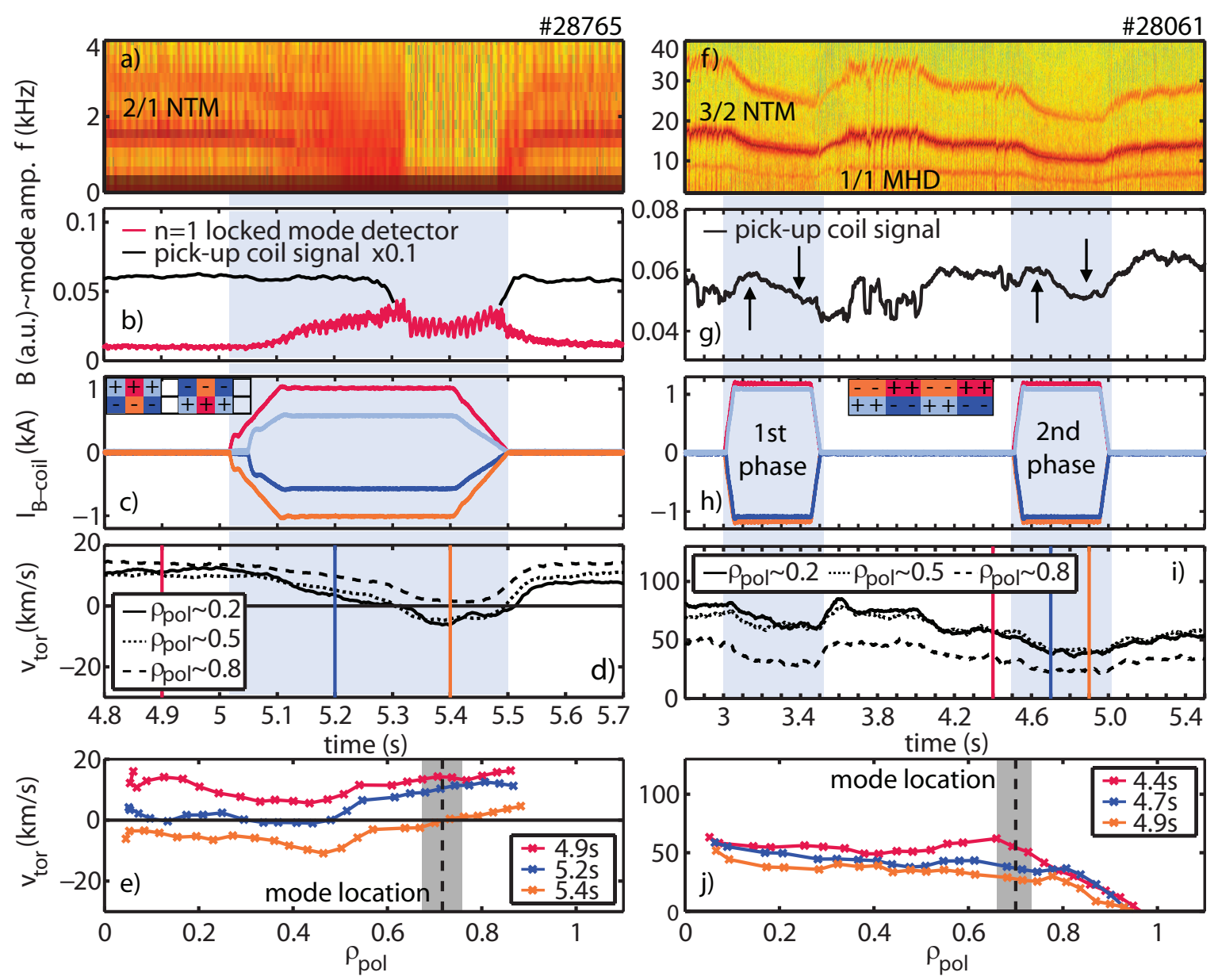

Figure 2. Overview of the two discharges which will be discussed. For each discharge a spectrogram of magnetic data (panel $(\mathrm{a})+(\mathrm{f})$ ), a qualitative signal for the mode amplitude (panel $(b)+(g)$ ), the current in one winding of the B-coils for four different coils (panel (c) and (h)), the time evolution of the toroidal plasma rotation from CXRS [39] at three radii (panel (d) + (i)) and profiles of the toroidal plasma rotation at three time points (panel (e) and $(\mathrm{j}))$ are shown.

\section{Experimental observation}

At AUG an influence of the MPs on NTMs is observed only in very few discharges so far. Two H-mode discharges are illustrated in figure 2. Each discharge corresponds to one of the two limits where the influence of the MPs on MHD is predicted to be strongest: low plasma rotation and medium plasma confinement, $\beta_{\mathrm{N}}=1.35$ or a dedicated plasma shape with low $q_{95}$, high $\beta_{\mathrm{N}}=2.1$, low density $n_{\mathrm{e}}=6 \cdot 10^{19} \mathrm{~m}^{-3}$ but substantial plasma rotation. The parameter $\beta_{\mathrm{N}}$ is defined as $\left(\beta_{\mathrm{pol}} a B_{\mathrm{pol}} / I_{\text {plasma }}\right)$, with $\beta_{\text {pol }}$ the ratio of kinetic pressure to poloidal magnetic pressure, $a$ the plasma minor radius, $B_{\text {pol }}$ the poloidal magnetic field and $I_{\text {plasma }}$ the plasma current. In the first case a $(2,1)$ NTM is 
slowed down and locks to the MP field, shown in figure 2 (a)-(e). In the second case a $(3,2)$ NTM is slowed down during two B-coil phases, as illustrated in figure 2 (f)-(j). In both discharges the plasma rotation (panel (d) and (i)) decreases together with the mode frequency (panel (a) and (f)) with a delay of around $50 \mathrm{~ms}$ after switching on the B-coils and it increases again with the same delay after ramping down the B-coils. In the locked mode discharge (\#28765) the plasma rotation decreases gradually and changes into counter-current direction for $\rho_{\text {pol }}<0.7$ during the locking phase, whereas at the island position the toroidal plasma rotation stays zero (panel (e)). In the braking case (\#28061) the toroidal rotation profile is almost flat from the mode location towards the core (panel (i) and $(\mathrm{j})$ ). In both discharges the plasma rotation decreases over the entire plasma radius.

Analysing the mode amplitudes (panel (b) and (g)) an other small difference of the two cases becomes obvious. Whereas the amplitude of the $(2,1)$ NTM, which is locking, is almost not changing, nor in the rotating neither in the locking phase, which means that the mode has a significant size even during locking, the $(3,2)$ NTM amplitude in terms of the perturbed radial magnetic field $B_{\mathrm{r}}(t)$, changes slightly correlated with the B-coils. The $(3,2) \mathrm{NTM}$, which is strongly rotating at around $15 \mathrm{kHz}$, is growing for about $150 \mathrm{~ms}$ then the mode amplitude is decreasing again. Despite the decrease of the island's amplitude a confinement reduction during the B-coil phases is observed correlated with a density pump out [8].

In the locking case an enhancement of the higher $m$ and $n$ harmonics, clearly correlated
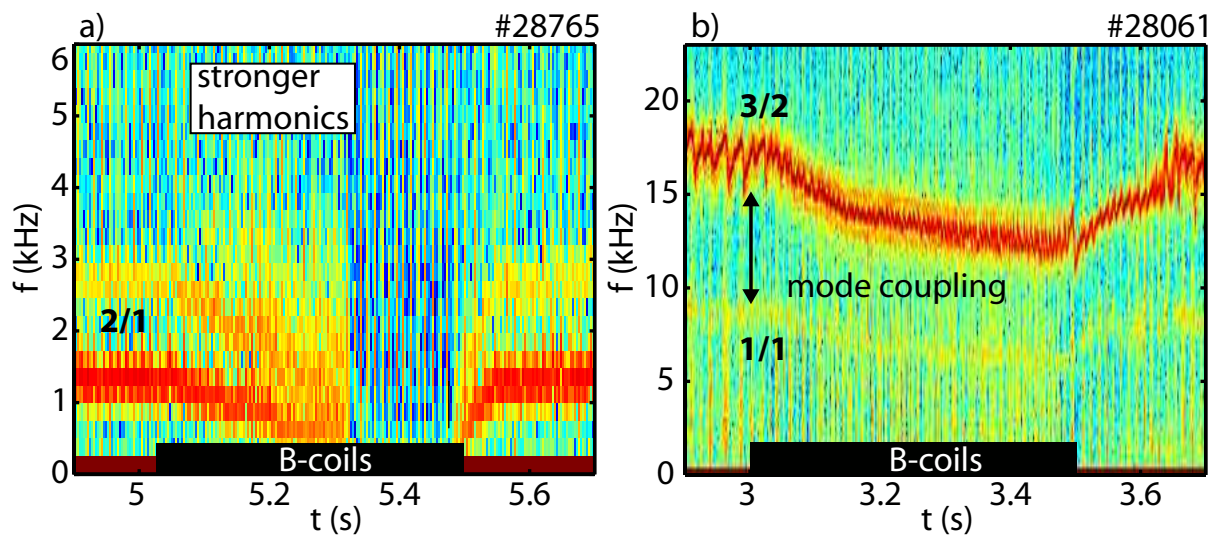

Figure 3. Spectrogram of one pick-up coil $\dot{B}_{\mathrm{r}}$ for discharge \#28765 (a) and \#28061 (b).

with the B-coils, can be observed, as shown in the spectrogram of one pick-up coil in figure 3 (a). This is caused by a distortion of the magnetic signals due to the influence of the MP field, as already reported in [40, 41, 42]. This irregularity is even visible directly in the evolution of the pick-up coil signals, shown in figure 4. Without MPs the characteristic signal $(\dot{B})$ of a mode is almost a sinusoid (panel (a)), whereas in the phases with B-coils a distortion is clearly visible, highlighted with the red arrows in panel (b) and (c). Shortly before the mode is locking (panel (c)) regions, where the mode is clearly 


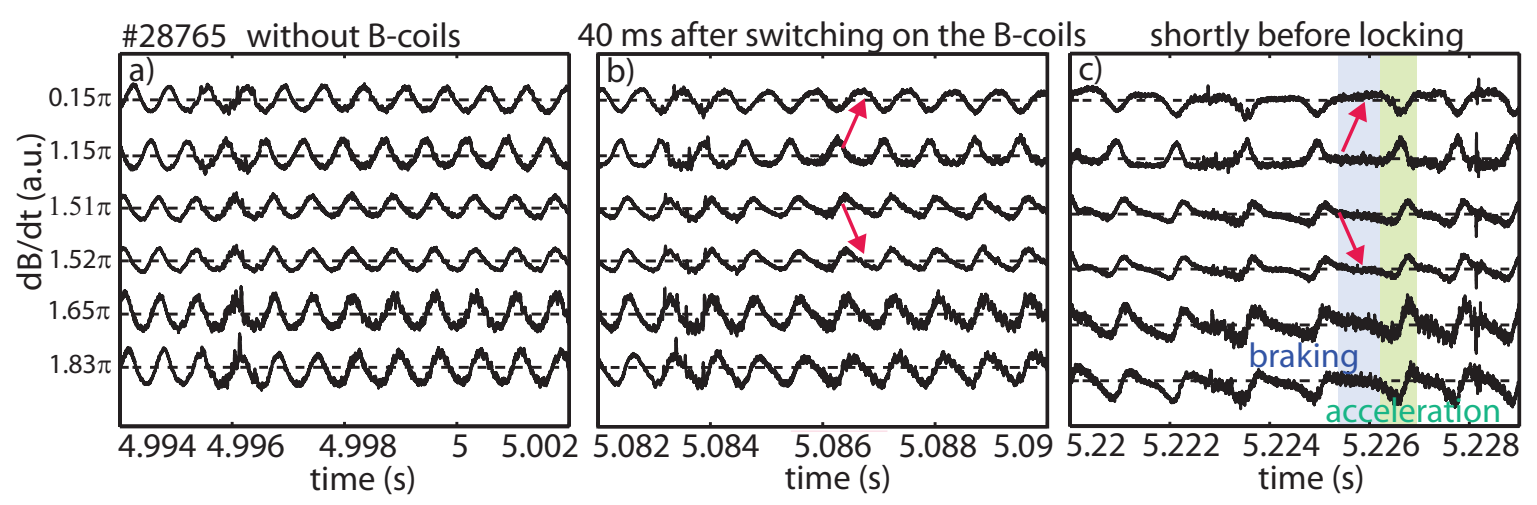

Figure 4. Time evolution of magnetic signals $\left(\dot{B}_{\mathrm{r}}\right)$ measured with pick-up coils at different toroidal positions for a $(2,1)$ NTM in $\# 28765$. Illustrated are (a) the unperturbed signals before switching on the B-coils, (b) slightly perturbed signals 30$40 \mathrm{~ms}$ after switching on the B-coils and (c) strongly perturbed signals shorty before mode locking appears. The arrows highlight phases where the increasing distortion can nicely be seen. The blue and green band in panel (c) highlight regions of mode braking and acceleration, respectively. The toroidal distribution of the pick-up coils is illustrated in figure 10 .

slowed down (blue) and regions where it is accelerated (green) are visible.

A small distortion is visible already shortly after switching on the B-coils, in a phase where the mode frequency has not changed significantly. Therefore, an increased influence of the resistive wall torque (compare equation (8)) can be excluded, which reveals that the distortion is caused by an impact of the B-coils. These variations have primarily an effect on the electron fluid. Additionally they are small and fast and hence, do not necessarily affect the whole main ion plasma rotation. Therefore they are not observed in the main ion plasma rotation shown in figure 2. Additionally the large error bars and the too slow time resolution of the rotation measurement hamper the detection of these rotation perturbations.

Due to the anhamonic structure of the mode also the ratio of the amplitude of the first and second harmonic is affected, which is illustrated in figure 5 (b), corresponding to the frequencies in figure 5 (a). For the locking case the ratio of second to first harmonic increases strongly during the B-coil phase. In the braking case (\#28061), neither a distortion of the magnetic signal nor an enhancement of the ratio $2^{\text {nd }} / 1^{\text {st }}$ harmonic can be observed. In figure 5 (d) a small effect might be superposed by strong variations in the island width and hence in the amplitude of the first harmonic. Nevertheless a clear correlation between the changes in the amplitude ratio and the B-coils is visible. A closer look into the spectrogram (figure 3 (b) of the braking case, reveals the $(3,2)$ NTM couples to the $n=2$ harmonic of a $1 / 1$ mode. The formation of this coupled mode system is likely to contribute to the strong influence of the B-coils on the mode frequency observed in this discharge even at those high rotation velocities. Additionally the coupling is consistent with the flat velocity profiles observed in experiment (figure $2(\mathrm{j}))$. 

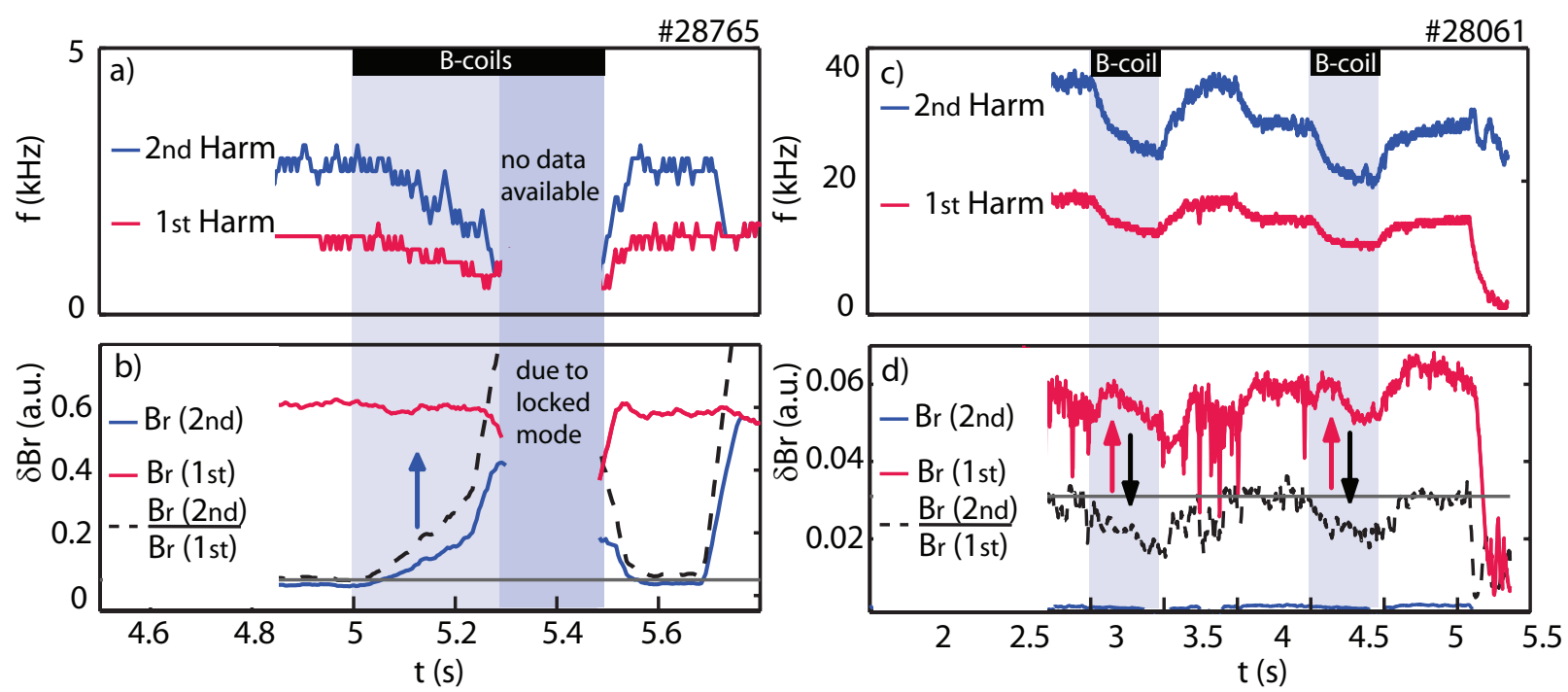

Figure 5. Frequency evolution of the $1^{\text {st }}$ and $2^{\text {nd }}$ harmonic during the B-coils phase for the locking (a) and the braking case (c). In panel (b) and (d) the amplitude of these two harmonics and their ratio is illustrated. In both cases changes in the ratio of the amplitude of $2^{\text {nd }}$ to $1^{\text {st }}$ harmonic are clearly correlated to the B-coils. Note that $B r(2 n d)$ in panel (d) is very small.

\subsection{Interpretation of experimental observation}

Most of those experimental observations can be explained by the influence of the resonant MP components on the mode. They lead to a modulation of the island width and frequency, which results in an anharmonic structure of the mode exactly as detected with the pick-up coils for the locking case. Since the influence of the resonant components decreases with increasing rotation frequency the effect is expected to be weaker in the braking case, where the mode rotation is substantial. Additionally, the different phasing of the modulation of the island amplitude and phase with respect to the mode rotation, according to equation (4) and (7) leads on average to a slowing down and a stabilisation of a rotating mode as well as to a destabilisation of a locked mode. This explains the strong decrease of the mode frequency observed in experiments. Nevertheless the predicted influence on the island stability is not observed in any of the discharges.

Thus, the experimental observations clearly show characteristics attributed to the resonant components of the perturbation field. However the fact that the island's amplitudes are only slightly affected, suggests that additional torques are acting, which do not influence the island stability at the resonant surface, but slow down the island rotation, like the NTV torque or resonant torques at other surfaces would do. This is in line with the presence of a small electron perpendicular velocity $v_{\perp, \mathrm{e}}$ for both discharges 
Table 1. Modelling parameter

\begin{tabular}{llllllll}
\hline Discharge & $r_{\text {res }}[\mathrm{m}]$ & $q$ & $q^{\prime}[1 / \mathrm{m}]$ & $B_{\text {tor }}[\mathrm{T}]$ & $\mathrm{C}$ & $W_{0}[\mathrm{~m}]$ & $W_{\text {vac }}[\mathrm{m}]$ \\
\hline 28061 braking & 0.27 & $(3,2)$ & 3.62 & 1.73 & 0.038 & 0.05 & 0.043 \\
28765 locking & 0.29 & $(2,1)$ & 7.44 & 2.5 & 0.0486 & 0.06 & 0.049 \\
\hline
\end{tabular}

which suggests that the influence of further resonant components in the linear phase is possible.

\section{Modelling of the island stability and rotation}

The interaction of a saturated, rotating magnetic island with the externally applied MPs can be calculated by solving the coupled equation system for the mode amplitude (eq. (2)) and phase (eq.(5)) [24] simultaneously. The initial island width $W_{0}$, which corresponds to the saturated island size in the unperturbed phase, is taken from the flattening of the $T_{\text {e }}$ profile measured at the low field side (LFS) of AUG. The width of the vacuum island $W_{\text {vac }}$ is determined from the vacuum approximation (fig. 1). An averaged vacuum island width on a flux surface is used, to take into account the elongated plasma shape and differences of high and low field side in the cylindric approach in equation (7) and (4). The equilibrium reconstruction provides $q$ profiles, which are consistent with the determination of the location of the resonant surface $r_{\text {res. }}$. All input parameters for the calculations are summarised in table 1 . The coefficient $A$ in equation (6) is adjusted in a way, that after switching off the B-coils the mode spins up to its natural frequency $\omega_{0}$ in the time observed in the experiment. This parameter $A$ in the presence of an island is in the order of $10^{-5} \mathrm{~kg} \mathrm{~m}^{2} / \mathrm{s}$ for the investigated discharges. Another way to determine the parameter $A$ is to assume, that the plasma rotation measured in the experiment is only caused by the applied NBI input torque. This method can only be applied when no mode is present due to the unknown torque this would cause. Knowing the rotation profile and the local input torque with $T_{\mathrm{NBI}}=-A / n<\Omega>$ the parameter $A$ can be determined. The estimated total NBI input torque is around 1-2 Nm per beam, depending on the injection angle. One example NBI input torque profile for the braking case (\#28061) is shown in figure 11 (c) together with the corresponding unperturbed rotation profile in figure 11 (a) in blue. According to these profiles this method delivers a parameter $A$ of around $0.4 \cdot 10^{-5} \mathrm{~kg} \mathrm{~m}{ }^{2} / \mathrm{s}$ which is in good agreement with the values of the first method listed in table 2. The corresponding momentum diffusion coefficient $D_{\phi}$ calculated later on is around $0.45 \mathrm{~m}^{2} / \mathrm{s}$.

Solving the EOM and the MRE including the effect of only the resonant magnetic perturbations leads to a slowing down of the island, which is far too small in both investigated cases. To match the frequency evolution of the modes in experiment two fit parameters, $c_{\mathrm{vac}}$ and $c_{\text {ext }}$, had to be introduced in equation (4) and (7). The vacuum island width is multiplied by the parameter $c_{\mathrm{vac}}$ to adjust the influence of the B-coils 
Table 2. Fit parameter

\begin{tabular}{llll}
\hline Discharge & $A\left[\mathrm{~kg} \mathrm{~m}^{2} / \mathrm{s}\right]$ & $c_{\mathrm{vac}}$ & $c_{\mathrm{ext}}$ \\
\hline 28061 braking $\left(1^{\text {st }} / 2^{\text {nd }}\right.$ phase $)$ & $0.5 \cdot 10^{-5} / 0.9 \cdot 10^{-5}$ & $4.06 / 4.0$ & $1 / 1$ \\
28765 locking & $1.1 \cdot 10^{-5}$ & 2.2 & 0.65 \\
\hline
\end{tabular}

in the equation of motion and the modified Rutherford equation. This is equal to introducing an effective vacuum island width $W_{\text {effective }}=W_{\mathrm{vac}} \cdot c_{\mathrm{vac}}$. By adjusting $c_{\mathrm{vac}}$ in the calculation a shielding or an amplification of the perturbation field compared with the vacuum case can be included. The second parameter $c_{\text {ext }}$ is used to diminish the modulation of the island width directly and hence the effect on the island width via a multiplication of $\Delta_{\text {ext }}$ with $c_{\text {ext }}$. All adjusted fit parameters are summarised in table 2 . In the calculation, the B-coils are switched on immediately and not ramped up as in the experiment. To correct this and the corresponding delay of the response of the mode during ramping up the B-coils, the B-coils in the modelling are switched on 0.05s later with respect to the experiment.

\subsection{Mode locking}

The modelling results for the island width and the rotation frequency for the locking case (\#28765) and the comparison to experiment are shown in figure 6. The
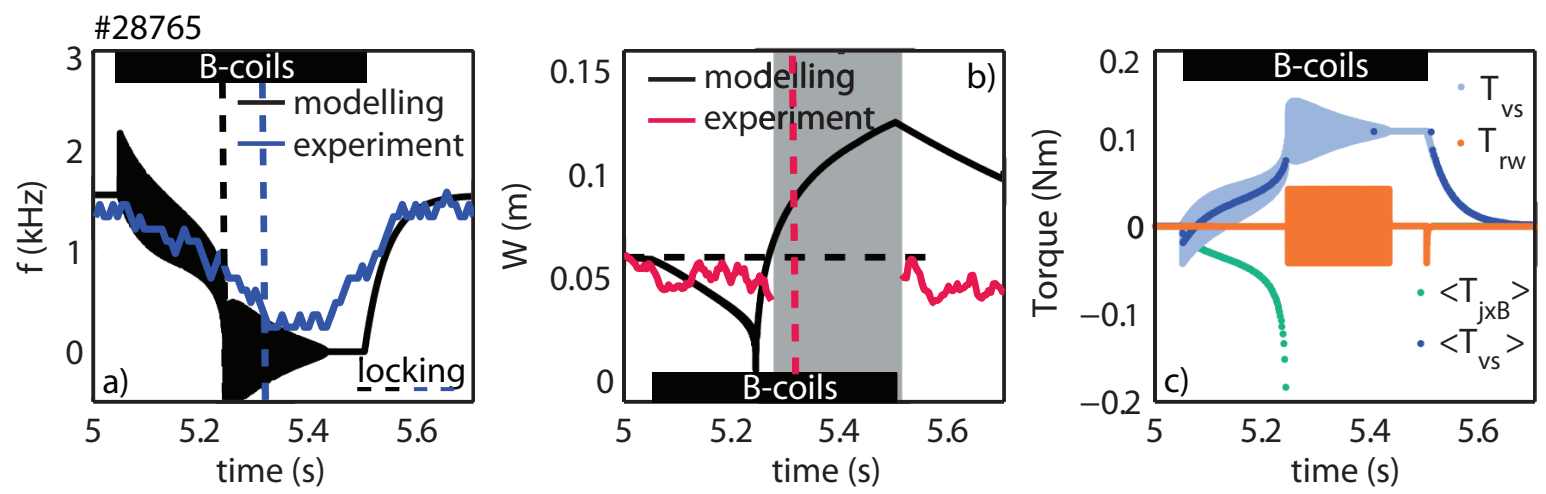

Figure 6. Calculated mode frequency (a) and island width (b) included the discussed assumptions each compared to the experimental quantities. In panel (b) the time interval, in which the mode amplitude can no longer be measured with the pick-up coils is highlighted in gray. This is not equal to mode stabilisation, the mode is still present but locked. In panel (c) the time evolution of the acting torques, where the viscous and the $(\vec{j} \times \vec{B})$ torque are averaged over $2 \pi$ are shown. The vertical dashed lines indicate the locking time in the calculation (black) and in experiment (coloured).

experimental mode frequency and island width evolution are determined from magnetic coil signals. The experimental island width from magnetic measurements are not absolutely calibrated therefore the evolution is scaled to match the unperturbed island width $W_{0}$ before switching on the B-coils. During the phase where the mode is locked 

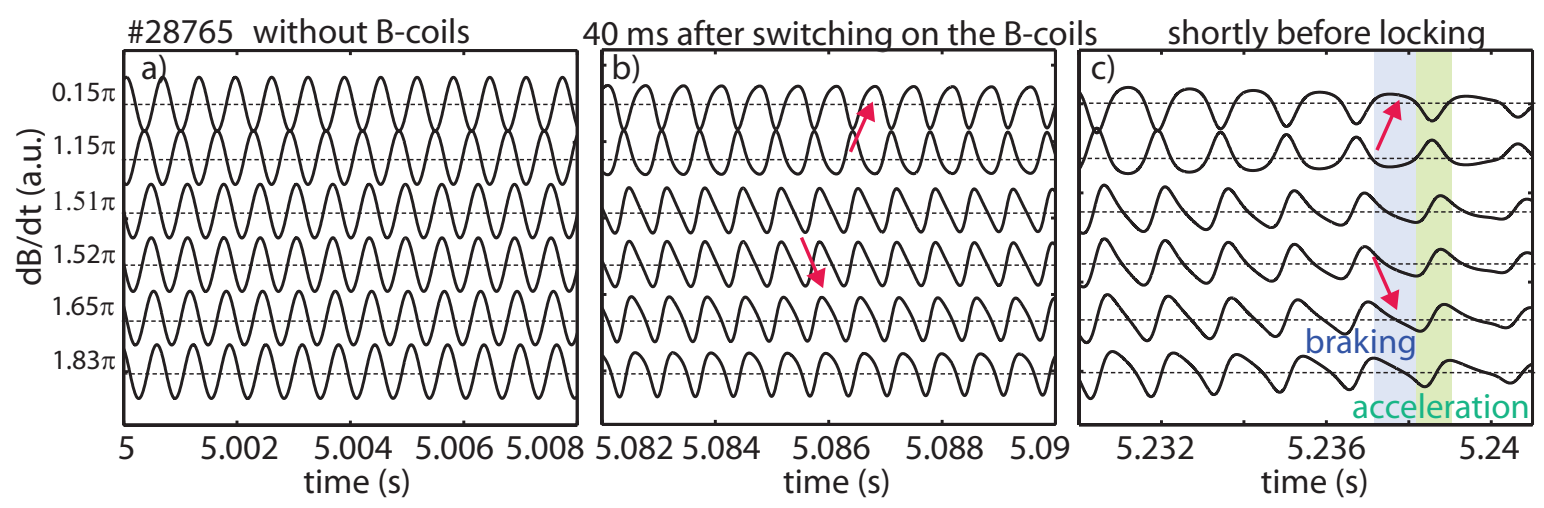

Figure 7. Simulated ballooning coil signals at different toroidal positions for the $(2,1)$ island similar to discharge \#28765). Illustrated are the unperturbed signals before switching on the B-coils (a), slightly perturbed signals 30-40 ms after switching on the B-coils (b) and strongly perturbed signals shorty before mode locking appears (c). The arrows highlight phases where the increasing distortion can nicely be seen. The blue and green band in (c) highlight regions of mode braking and acceleration respectively.

to the wall the pick-up coils (red signal) are not able to detect the changes in the island amplitude anymore. This does not correspond to a stabilisation of the island, compare figure 2. The mode is still present but not rotating anymore.

The best agreement of experiment and calculation for the frequency evolution is achieved by, increasing the vacuum island size by a factor of 2.2 . This enhancement additionally leads to a stabilisation of the island before it is locking. Since this is not observed in experiment the influence of the B-coils on the island width evolution had to be decreased directly by a factor $\left(c_{\text {ext }}\right)$ of 0.65 .

The island is still strongly stabilised in the calculation long before mode locking appears and grows strongly during the locking phase, which is not in agreement with the experiment, where the island width almost stays constant. The fact that the mode amplitude is not affected while slowly rotating could hint towards the observations made in [43]. A regime was found at low rotation where screening effects are observed numerically for slowly rotating islands due to magnetic curvature effects, similar to the MP field screening at high rotation.

In the calculation the actual locking of the mode can only be reproduced by including the resistive wall term. However by definition (eq. 8) $T_{\phi, \mathrm{rw}}$ depends on $W^{4}$, hence it will grow rapidly even at small rotation velocities using the large calculated island width $W$. Therefore the restoring viscous torque will not be able to unlock the island after switching off the B-coils. Since unlocking is observed in experiment and also the island width is not increasing, in the calculation the dependence on $W$ is replaced by $T_{\phi, \text { rw }}\left(W_{0}\right)$, which is in better agreement with experiment. Additionally the mode is locking earlier in the modelling than in experiment.

All these assumptions, which have to be made, reveal that the effect on the island width is overestimated, whereas the effect on the island frequency seems to be amplified compared to the vacuum phase. One solution would be that additional torques induced 
by the MP field exist which slow down the island but does not influence the island amplitude. However the modulation of the mode amplitude and phase due to the resonant MP components is clearly reproduced in the modelling. The synthetic pick-up coil signals show a clear distortion (figure 7) comparable to the experiment. Also the resulting enhancement of the higher harmonics in the spectrogram during the B-coil phase is reproduced, shown in figure 8 (a).
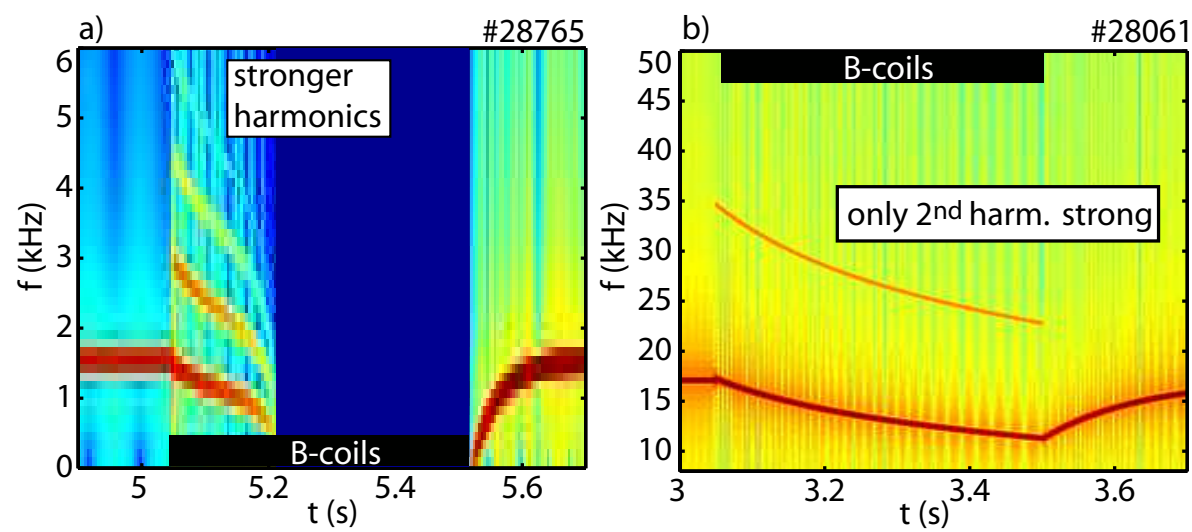

Figure 8. Spectrogram calculated from one single simulated magnetic pick-up coil signal, as shown in figure 7 for the locking (\#28765) (a) and the braking case (\#28061) (b).

\subsection{Mode braking}

The modelling results for the braking case (\#28061) for both B-coil phases are shown in figure 9, where the mode frequency, the island width and the acting torques are illustrated. To match the experimental mode frequency evolution (panel (a)) $W_{\text {vac }}$ had to be increased by a factor of 4 in both B-coils phases. The impact on the island width evolution directly was unchanged in this case, hence the second fit parameter $c_{\text {ext }}$ is one. In the simulation the destabilisation in the first part of the B-coil phases can not be reproduced (figure $9(\mathrm{~b})$ ) , as it is not included in the model, but the trend of stabilising the island in the second part of the B-coil phases can be modelled. However the stabilising effect is slightly overestimated. The modelling results in a maximal resonant $(\vec{j} \times \vec{B})$ torque of around $0.1 \mathrm{Nm}$ (panel (c)). The resistive wall torque can be neglected for such high mode frequencies, as visible from panel (c). Similar to the experiment for the braking case no big distortion of the simulated magnetic signal is visible. A small distortion can be detected in figure 8 (b) due to a visible enhancement of only the second harmonic during the B-coil phase.

\section{Error field direction}

From the comparison of the simulated and experimental pick-up coil signals the direction of the resulting error field for the locking case can be determined, which includes the 

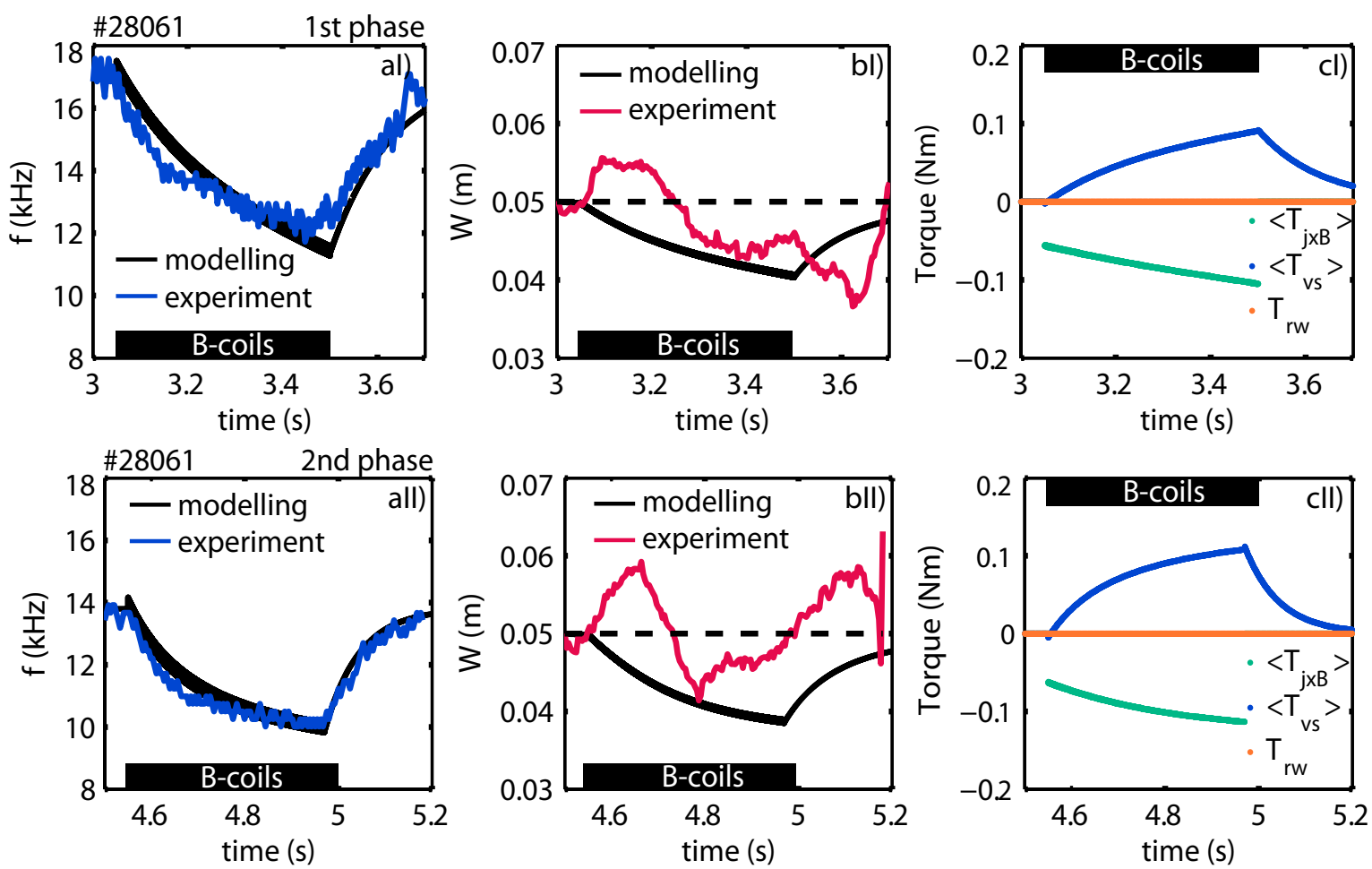

Figure 9. Calculated mode frequency (a) and island width (b) each compared to the experimental quantities. In panel (c) the time evolution of the acting torques, where the viscous and the $(\vec{j} \times \vec{B})$ torque are averaged over $2 \pi$ are shown. On the top the first B-coil phase and on the bottom the second B-coil phase is illustrated.

external field but also other internal field contributions. The total resulting error field is responsible for the distortion of the mode signal. Its orientation in the toroidal plane determines the different impact on the mode depending on the toroidal location, like for example the braking and acceleration in different toroidal regions. By rotating the error field in the modelling until the modelled signal structure matches the experimental one for every coil position, the direction of the resulting error field can be determined [36]. The different positions of the coils used for this analysis are illustrated in figure 10 . The resulting error field direction can then be compared to the position of the locked mode and the position of the $(2,1)$ vacuum field direction, which is assumed have the dominating impact on the mode in the modelling in section 4.1.

The position of the locked mode in experiment is determined with saddle coils located at the high field side [44]. The $(2,1)$ vacuum field direction is identified from the position of the $(2,1)$ vacuum island. The resulting error field direction and the experimental locked mode position in the toroidal plane are illustrated in figure 10. Additionally the position of the $(2,1)$ vacuum island is indicated. The experimental locked mode location (blue) is shifted about $40^{\circ}$ in counter-current direction compared to the error field direction determined from the modelling (orange). The island does not lock in the minimum of the error field potential $(\sim-\cos (\Delta \phi))$ at $\Delta \phi=0$ but is slightly driven, "up the hill". This is caused by the driving torque due to the main plasma rotation and the viscous coupling 
Toroidal cross section

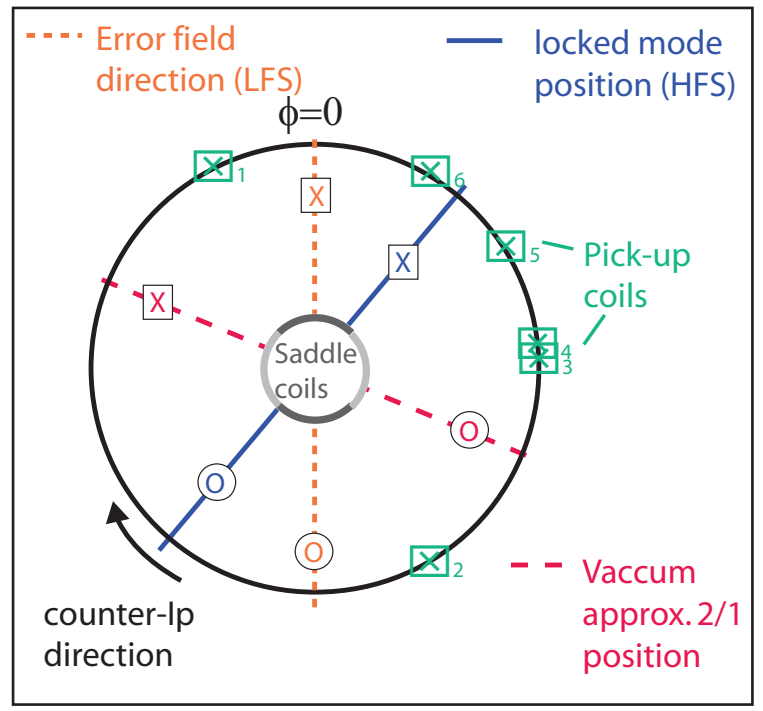

Figure 10. Location of the locked mode measured with the locked mode detector (blue) compared to the error field direction (orange) and the position of the vacuum island (red) in the toroidal plain. Additionally, the position of the pick-up coils (dark green) used in figure 4 and 7 are indicated.

to the island. Therefore a phase shift of less than $90^{\circ}$ in counter-current direction on the basis of a counter rotating plasma (compare figure 2 (e)) is reasonable. However the $(2,1)$ vacuum field location (red) does not agree with the determined error field direction. This implies that not only the $(2,1)$ component of the MP field contributes to the slowing down and locking of the NTM but also other resonant components and the intrinsic error field. This means that a combination of resonant contributions at different resonant surfaces, caused by the external or intrinsic error field, act on the plasma. These torques together lead to a slowing down of the plasma and concomitant to a slowing down of the island. The interplay of these torques could lead to a deviation of the locking position from the single $(2,1)$ vacuum field direction.

\section{Reconstruction of the entire rotation profile during RMPs}

In order to investigate the influence of different combination of torques, also at different rational surfaces, the evolution of the entire rotation profile (fig. 11 (b)) is modelled for the braking case. The equation of motion is solved accounting for the radial dependence of all torques. The modelling results can then be compared to the experimentally observed evolution. For this analysis the viscous torque $T_{\mathrm{vs}}$ with a constant momentum diffusion coefficient, the resonant $(\vec{j} \times \vec{B})$ torque $T_{\mathrm{j} \times \mathrm{B}}$, the non-resonant NTV torque $T_{\mathrm{NTV}}$ and the NBI input torque $T_{\mathrm{NBI}}$ with their radial dependences are included in the equation of motion (5). Additionally, the coupling of the $(2,2)$ component of the $(1,1)$ mode and the $(3,2) \mathrm{NTM}$ via $T_{\mathrm{cpl}}$ observed in experiment are quantitatively taken into account [37]. In figure 11 the experimental rotation profiles from CXRS (panel (a)), the 
torques used for the modelling (panel (b)), and the modelled rotation profiles (panel (c) and (d)) are shown.
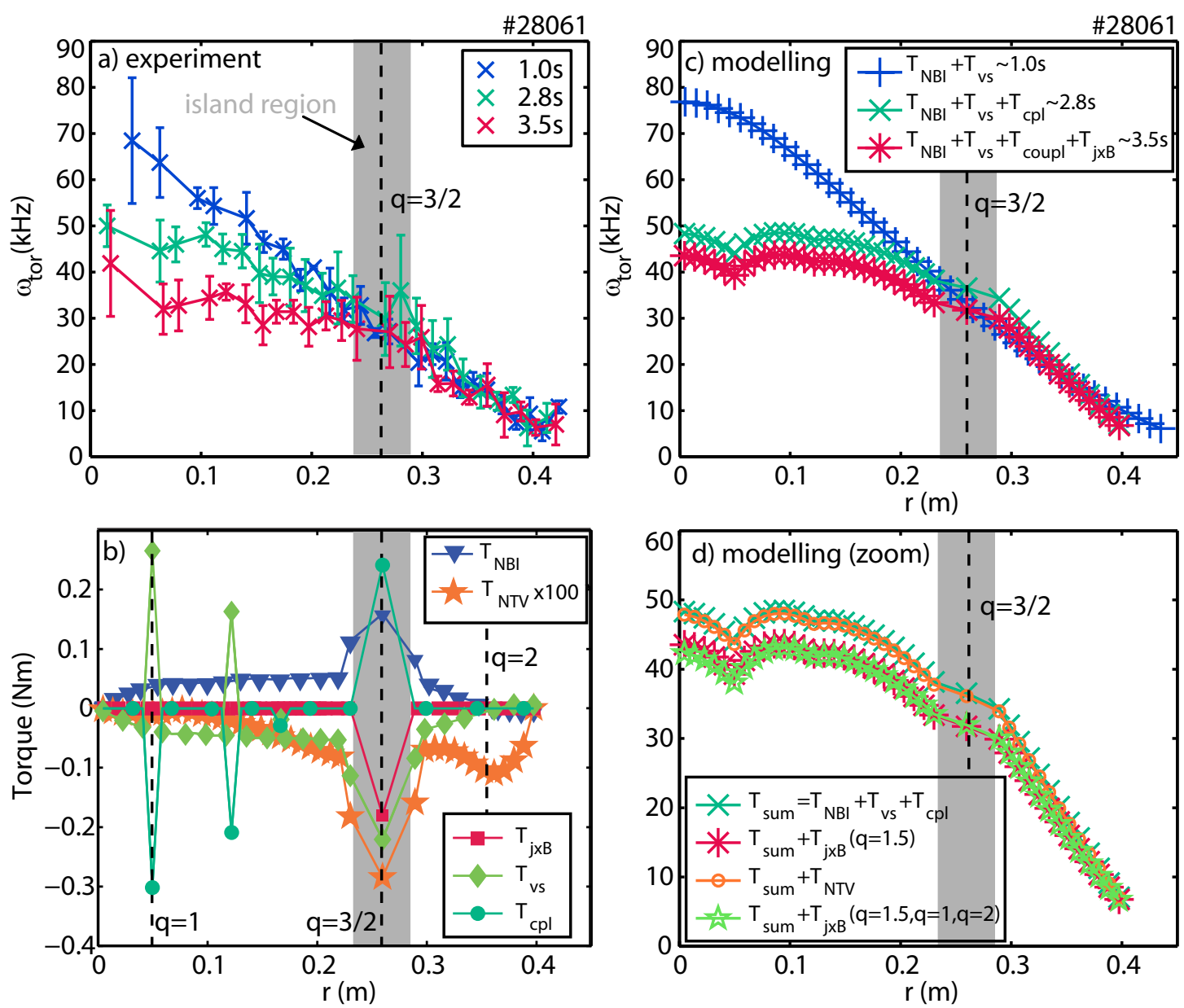

Figure 11. In (a) the experimental rotation profiles are shown at the beginning of the discharge $(1.0 \mathrm{~s})$ where no modes are present in dark blue, before switching on the B-coils $(2.8 \mathrm{~s})$ in dark green and at the end of the B-coil phase $(3.5 \mathrm{~s})$ in red. In (c) the corresponding modelled rotation profiles are shown, where the colour coding corresponds to (a). All torques used to achieve the red profile in (c) are shown in (b): the NBI torque (dark blue), the viscous torque (light green), the coupling torque (dark green) and the resonant torque at the mode surface (red). Additionally the NTV torque profile calculated for $3.0 \mathrm{~s}$ at the beginning of the B-coil phase is shown in orange. Note that the NTV profile in (b) is scaled by a factor of 100. (d) Shows the small influence of the NTV torque (orange) on the unperturbed rotation profile (dark green) compared to the influence of the resonant $(\vec{j} \times \vec{B})$ torque, which is almost the same exerting the torque at only one (red profile) or different radial positions (light green).

First of all the rotation profile at the beginning of the discharge without any big mode and external perturbation being present is simulated (figure 11 (c) in blue). This is done by balancing the toroidal component of $T_{\mathrm{NBI}}$ and $T_{\mathrm{vs}}$ by adjusting the momentum diffusion coefficient $D_{\phi}$. The adjustment of the modelled profile (panel (c) blue) to the experimental profile yields a $D_{\phi}$ of around $0.4 \mathrm{~m}^{2} / \mathrm{s}$. Example torque profiles of $T_{\mathrm{NBI}}$ and 
$T_{\mathrm{vs}}$ corresponding to a later time point $(3.5 \mathrm{~s})$ are shown in panel (b) in dark blue and light green, respectively. The NBI deposition profile is taken from TRANSP simulations [45]. In the next step the dark green rotation profile (panel (a)), present shortly before the B-coils are switched on at $2.8 \mathrm{~s}$ is reproduced. At this time point already the $(1,1)$ and the $(3,2)$ NTM are present. To reproduce this flat profile mode coupling is included qualitatively, by introducing opposite torques $T_{\mathrm{cpl}}$ at the $q=1, q=3 / 2$ surface and inbetween. These local torques are shown in dark green in panel (b) [37]. These torques are adjusted manually to reproduce the profile flattening observed in experiment. The modelled profile is shown in dark green in panel (c).

Starting from the dark green rotation profile, where $T_{\phi, \mathrm{vs}}$ and $T_{\mathrm{cpl}}$ are now adjusted for, the influence of the calculated resonant $(\vec{j} \times \vec{B})$ and non-resonant (NTV) torques on the rotation profile can be investigated and compared to the experiment. To reproduce the decrease of the rotation profile observed in experiment due to the B-coils (dark green to red profile panel (a)), a local $(\vec{j} \times \vec{B})$ torque of $0.18 \mathrm{Nm}$ at the $q=3 / 2$ surface has to be introduced. This is in good agreement with the $0.1 \mathrm{Nm}$ calculated in section 4.2. The rotation decreases globally in the modelling, also when exerting a local torque, which agrees with the experimental profile evolution. This local torque is shown in red in panel (b). Almost the same rotation profile can be achieved, exerting smaller torques at different radial positions. For example exerting a torque of $0.07 \mathrm{Nm}$ at each of the $q=1, q=3 / 2$ and $q=2$ surfaces, delivers almost the same rotation profile than in the previous case (light green profile panel $(d)$ ). In the modelling of the entire rotation profile also the NTV torque profile, according to [32] and shown in panel (b) in orange, can be included. Including $T_{\mathrm{NTV}}$ in the torque balance equation results in a decrease of rotation of around $0.4 \mathrm{kHz}$ (orange profile panel $(\mathrm{d})$ ). This corresponds to around $8 \%$ of the total decrease of rotation observed in the experiment $(\approx 5 \mathrm{kHz})$. This confirms that the NTV is negligibly small.

Therefore, the rotation damping and the concomitant slowing down of the NTM in the investigated discharges, can be assumed to be dominantly induced by the resonant perturbation field components. Additionally, it is shown that in fact the same decrease of rotation, also at a specific resonant surface, can be achieved when exerting several smaller torques at different resonant surfaces.

\section{Summary and Conclusions}

The effect of MP fields on NTMs has been investigated in the AUG tokamak. Mode braking and locking due to the MPs is observed, as well as the spinning up of the mode when switching off the B-coils. In the investigated discharges the mode frequency as well as the global plasma rotation both decreases. In contrast, the mean mode amplitude is only slightly influenced. In the locking case a strong modulation of the island width and frequency is observed, directly via a distortion of the pick-up coil signals evolution and indirectly via the enhancement of higher harmonics in the spectrograms. This is less pronounced in the braking case, due to a higher mode rotation. 
The NTV torque caused by the non-resonant MP components has been calculated accounting for the real perturbation field and plasma geometry. The calculation showed that the NTV torque is small and that its influence on the plasma rotation is negligible. Hence, the resonant MP components are dominant. Accounting only for the influence of the resonant MP field components the mode braking and locking, as well as the modulation of the island width, can be reproduced by the modelling. However, in both modelling cases the influence of the perturbation on the mode had to be increased, compared to the calculated vacuum approximations, to match the experimental frequency evolution. As a consequence, the effect on the island width is overestimated. This could be solved by including an additional torque in the modelling, which decreases the mode frequency but does not influence the island stability. Especially in the braking case, the presence of a $(1,1)$ mode with the corresponding large resonant MP field component at the $q=1$ surface reveals, that the influence of the $(2,2)$ resonant component seems not to be insignificant.

For the locking case, the direction of the resulting error field, which is responsible for the modulation of the island width and frequency depending on the phase of the mode, could be determined from the modelling. The error field direction is in good agreement with the actual experimental locked mode position but it does not coincide with the $(2,1)$ vacuum island position. This leads to the assumption that the sum and interaction of different contributions, internal or external, influence the slowing down and locking of the mode and not only one dominant external component.

The modelling of the entire rotation profile for the braking case, accounting for the radial dependence of all torques and a constant momentum diffusion coefficient, reveals that a combination of smaller resonant torques at different surfaces can lead to the same rotation decrease at the mode surface as a single large torque at the NTM surface. This means that it is possible that smaller $(\vec{j} \times \vec{B})$ torques at several surfaces sum up to a total torque, which has the same effect as one large local one. This leads to a smaller influence on the mode stability at one specific resonant surface. This can additionally be confirmed with the fact that also in experiment (figure 1) vacuum islands induced by the external MP field appear at several different surfaces. Most of them are smaller than the dominating one at the position of the NTM but in sum they seem to have a significant impact and as show in [46] coupling between higher harmonics with same toroidal mode number can lead to a significantly larger perturbation field at the resonant surfaces compared to the vacuum values. This is true for the mode surface but also for all other resonant surfaces inside the plasma. However, this is beyond the scope of the minimised model used within this paper.

Additionally toroidal curvature effects [43] could play a role. At low rotation, like in the locked mode case, it is predicted that these effects lead to an enhancement of the MP field amplitude and a more global than local torque input due to enhanced sideband harmonics. Beside these effects in both discharges, the torque induced by the loss of fast ions, which is strongly enhanced during the B-coil phases $[9,10]$ could be an additional contribution. In addition also the cylindric approximation of the resonant effects and 
their parametrisation via the vacuum approximation is responsible for deviations from the experimental observations.

Concluding, the NTV caused by the MP field seems to be small at AUG but the resonant torques at different resonant surfaces act together as a global resonant torque that is responsible for the rotation damping. To describe the evolution of a single magnetic island accounting for the influence of the resonant MPs, all islands present in the plasma and the impact of all resonant contributions at several surfaces have to be taken into account or effects which lead to non-local resonant torques have to be included.

\section{Acknowledgements}

The authors thank T. Lunt for the support by calculating the vacuum field approximation.

This work has been carried out within the framework of the EUROfusion Consortium and has received funding from the European Unions Horizon 2020 research and innovation programme under grant agreement number 633053. The views and opinions expressed herein do not necessarily reflect those of the European Commission. 


\section{References}

[1] HENDER, T., FITZPATRICK, R., MORRIS, A., et al., Nuclear Fusion 32 (1992) 2091.

[2] SUTTROP, W., EICH, T., FUCHS, J. C., et al., Phys. Rev. Lett. 106 (2011) 225004.

[3] EVAns, T. E., MOYeR, R. A., Thomas, P. R., et al., Phys. Rev. Lett. 92 (2004) 235003.

[4] STRAit, E. J., BIALEK, J. M., BOGATU, I. N., et al., Physics of Plasmas 11 (2004) 2505.

[5] HU, Q., YU, Q., RAO, B., et al., Nuclear Fusion 52 (2012) 083011.

[6] EVAns, T., FEnStermacheR, M., MOYER, R., et al., Nuclear Fusion 48 (2008) 024002.

[7] SCHMitZ, O., EVAnS, T. E., FENSTERMACHER, M. E., et al., Plasma Physics and Controlled Fusion 50 (2008) 124029.

[8] MORDIJCK, S., MOYER, R. A., KIRK, A., et al., Plasma Physics and Controlled Fusion 53 (2011) 122001.

[9] GARCIA-MUNOZ, M., AKASlOMPOlO, S., ASUNTA, O., et al., Nuclear Fusion 53 (2013) 123008.

[10] Garcia-Munoz, M., AKASlompolo, S., DE MARne, P., et al., Plasma Physics and Controlled Fusion 55 (2013) 124014.

[11] KOSLOWSKI, H., LIANG, Y., KRMER-FleCKEN, A., et al., Nuclear Fusion 46 (2006) L1.

[12] YU, Q., GÜNTER, S., KIKUCHI, Y., and FINKEN, K., Nuclear Fusion 48 (2008) 024007.

[13] COle, A. J., HeGnA, C. C., and CAllen, J. D., Phys. Rev. Lett. 99 (2007) 065001.

[14] SUTTROP, W., GRUBER, O., GÜNTER, S., et al., Fusion Engineering and Design 84 (2009) 290, Proceedings of the 25th Symposium on Fusion Technology (Rostock, Germany, September 15th - 19th 2008).

[15] RUTHERFORD, P. H., Physics of Fluids 16 (1973) 1903.

[16] SAUter, O., ANGIONI, C., and LIN-LIU, Y. R., Physics of Plasmas 6 (1999) 2834.

[17] SAUter, O., BUtTery, R. J., FELTON, R., et al., Plasma Physics and Controlled Fusion 44 (2002) 1999.

[18] FITZPATRICK, R., Physics of Plasmas 2 (1995) 825.

[19] Wilson, H. R., CONnOR, J. W., HASTIE, R. J., and HEGnA, C. C., Physics of Plasmas 3 (1996) 248.

[20] GLasser, A. H., Greene, J. M., and JOhnson, J. L., Physics of Fluids 18 (1975) 875.

[21] Glasser, A. H., Greene, J. M., and Johnson, J. L., Physics of Fluids 19 (1976) 567.

[22] LÜtJens, H., LUCIANI, J.-F., and GARBET, X., Physics of Plasmas 8 (2001) 4267.

[23] Kotschenreuther, M., HAZELtine, R. D., and MORRISON, P. J., Physics of Fluids 28 (1985) 294.

[24] FITZPATRICK, R., Nuclear Fusion 33 (1993) 1049.

[25] SHAing, K. C., Physics of Plasmas 10 (2003) 1443.

[26] CAllen, J., COlE, A., and HEGNA, C., Nuclear Fusion 49 (2009) 085021.

[27] FITZPATRICK, R., Plasma Physics and Controlled Fusion 54 (2012) 094002.

[28] Garofalo, A. M., BurRell, K. H., DeBOO, J. C., et al., Phys. Rev. Lett. 101 (2008) 195005.

[29] Garofalo, A. M., SOlOmon, W. M., LANCTOT, M., et al., Physics of Plasmas 16 (2009) 056119 .

[30] SUN, Y., LIANG, Y., SHAING, K., et al., Nuclear Fusion 52 (2012) 083007.

[31] ZHU, W., SABBAGH, S. A., BELL, R. E., et al., Phys. Rev. Lett. 96 (2006) 225002.

[32] COLE, A., HEGNA, C., and CALLEN, J., Low collisionality neoclassical toroidal viscosity in tokamaks and quasi-symmetric stellerators using integral-truncation technique, Technical report, UW CPTC Report 08-8, Wisconsin, 2009, http://www.cptc.wisc.edu/reports/UWCPTC_08-8.pdf.

[33] Militello, F., HAStie, R. J., and PORCElli, F., Physics of Plasmas (1994-present) 13 (2006) .

[34] SMolyakov, A. I., Hirose, A., LAZZARO, E., RE, G. B., and CAllen, J. D., Physics of 
Plasmas (1994-present) 2 (1995) 1581.

[35] STIX, T. H., Physics of Fluids 16 (1973) 1260.

[36] ZOHM, H., KAllenBaCh, A., BRUhns, H., FUSSMANN, G., and KLÜBER, O., EPL (Europhysics Letters) 11 (1990) 745.

[37] CLASSEN, I., Imaging and Control of Magnetic Islands in Tokamaks, PhD thesis, Technische Universiteit Eindhoven, 2007.

[38] FitZPATRICK, R. and WAELBROECK, F. L., Physics of Plasmas (1994-present) 7 (2000) 4983.

[39] VIEZZER, E., PÜTTERICH, T., DUX, R., McDERMotT, R. M., and the ASDEX Upgrade Team, Review of Scientific Instruments 83 (2012) 103501.

[40] IVANOV, N., KAKURIN, A., and ORLOVSKIY, I., Irregularity of the magnetic island rotation under external helical magnetic perturbation in t-10 tokamak, 2005, Proceedings of the $32^{\text {th }}$ European Physical Society Conference on Plasma Physics, Tarragona, 2005, No. P-5.068, http://epsppd.epfl.ch/Tarragona/pdf/P5_068.pdf.

[41] IVANOV, N., KAKURIN, A., and KONOVALOV, S., PAS\&T/TF 36 (2013) 55.

[42] HU, Q., RAO, B., YU, Q., et al., Physics of Plasmas (1994-present) 20 (2013) .

[43] LIU, Y., CONNOR, J. W., COWLEY, S. C., et al., Physics of Plasmas (1994-present) 19 (2012)

[44] MARASCHEK, M., FIETZ, S., GUDE, A., et al., Measurement and impact of the $\mathrm{n}=1$ intrinsic error field at asdex upgrade, 2013, Proceedings of the $40^{\text {th }}$ European Physical Society Conference on Plasma Physics, Espoo, Finland, 2013, No. MCF/P4.127, http://eps2013.aalto.fi/?page=proceedings.

[45] PANKIN, A., MCCUNE, D., and ANDRE, R., Computer Physics Communications 159 (2004) 157.

[46] PARK, J.-K., SChAfFeR, M. J., MENARD, J. E., and BOOZER, A. H., Phys. Rev. Lett. 99 (2007) 195003. 